Wasser verdünnt, mittelst einer Schlundsonde in den Magen gespritzt und nach Verlauf einer Stunde schon Leber, Herz, Sceletmuskeln auf Glykogen untersucht.

Mit einer einzigen Ausnahme fanden sich jetzt bei den Zuckerthieren durch Jodreaction schon recht deutlich erkennbare Mengen von Glykogen in der Leber, einige Male auch im Herzen, nie in den Sceletmuskeln. Auch nach der Glyceringabe war mehrfach Glykogen in deutlichster Weise wiederum in der Leber nachweisbar, fehlte aber jetzt sowohl im Herzen wie in den Sceletmuskeln.

Also genügen in der That schon kleine Mengen von Zucker wie von Glycerin, um schon nach kurzer Zeit wiederum Glykogen in einer vorher sicher vollkommen glykogen freien Leber erscheinen zu lassen - ein Resultat, dessen Sicherheit wir gegenuiber jenen negativen Ergebnissen einer durchaus ähnlichen Versuchsreihe Bernard's') nur um so mehr betonen mïssen; in jenen Fällen folgte eben die Tödtung des Thieres erst volle sechs Stunden nach der Injection, die zu lange Dauer der Versuchszeit dürfte allein schon den Misserfolg verschuldet haben.

(Aus dem physiologischen Laboratorium in Zürich.)

\title{
Die Erregbarkeit der Schweissdrüsen als Function ihrer Temperatur.
}

$$
\text { Von. }
$$

\section{B. Luchsinger.}

Ganz allgemein ist jedes lebende Wesen zu seiner Entwickelung wie zu seiner Thätigkeit an bestimmte günstigste Temperaturen gebunden, wird dessen physiologische Leistung sowohl durch Erniedrigung wie durch weitere Steigerung derselben gleicherweise herabgesetzt, ja völlig gehemmt.

Hedysarum gyrans beginnt erst iiber $22^{\circ} \mathrm{C}$. das reizende

1) Cl. Bernard, leçons sur le diabète, Paris 1877. 
Spiel seiner Blättchen, mit steigender Erwärmung wird dasselbe immer lebhafter, bis die Temperatur auf c. $45^{0}$ steht; steigt dieselbe aber noch weiter, so büsst die Bewegung an Lebendigkeit ein und hört endlich ganz auf bei c. $48^{\circ}$, um bei bald folgendem Absinken der Hitze an Intensität wieder mehr und mehr zu gewinnen.

Das Centralnervensystem des Frosches nimmt ron Eisestemperatur bis gegen $35^{\circ}$ fortwährend an Erregbarkeit zu, nur wenige Grade höher erwärmt (380) stellt es jedoch seine Functionen gänzlich ein, und nimmt dieselben erst wieder auf, sobald die Temperatur wieder entsprechend abgesunken.

Nach Anschauungen, wie solche noch zuletzt in überzeugendster Art Pflüger (diess Archiv X. 1875) entwickelt hat, ist eine Verlangsamung der Lebensprocesse durch zu niedrige Temperaturen geradezu selbstverständlich; weniger sorgfältig untersucht und desshalb auch weniger discutirt ist der nur vorübergehende, hemmende Einfluss zu starker Erwärmung. Irre ich nicht, so dürfte es sich hier um eine noch restituirbare Vorbereitung jener Gerinnungen handeln, welche nur wenige Grade höher in allen protoplasmatischen Massen eintreten und dadurch dauernd den Lebenserscheinungen ein Ende setzen.

Nur höchst selten erst haben sich bislang die Gewebe des Warmblïters einer einschlagenden Untersuchung erfreut. Ihrer oberflächlichen Lage halber eignen sich die Schweissdrüsen vortrefflich als Versuchsobject. -

a) Versuche über den Einfluss der Kälte auf die Erregbarkeit der Schweissdrüsen. Schon bei Gelegenheit einiger zusammen mit Hrn. Trümpy ${ }^{\top}$ ) angestellten Versuche, in welchen der plex. brachialis des Menschen mit tetanisirenden Strömen gereizt wurde, um hernach den Schweiss der Hand auf seine Reaction prüfen zu können, machten wir die Erfahrung, dass die Secretion trotz gleichen Reizes gleichwohl sehr grosse Schwankungen zeigen kann, Ja es konnte sich ereignen, dass die Secretion öfters geradezu ausblieb, oder doch nur in sehr geringen Mengen erschien. Bei kaltem Wetter traf diess gemeiniglich zu.

Nicht selten (besonders zur Winterszeit) sah ich im Verlauf anderweitiger, länger dauernder Versuche an Katzen, offenbar Hand in Hand mit allgemeiner Abkühlung des Thieres Nervenreizungen für die Erregung von Schweiss erfolglos werden. Stets stellte sich jedoch die Erregbarkeit in solchen Fällen wieder ein, sowie nur die Pfote durch Eintauchen in warmes Wasser wieder etwas höhere Temperatur erlangte.

1) Vgl. unten pag. 494 u. flgd. 
Werden beide Hüftnerven einer Katze durchschnitten, mit Ludwigschen Elektroden armirt, die eine Hinterpfote für einige Zeit (ca. 10 Min.) in Eiswasser, die andere in Wasser von ca. $30^{\circ} \mathrm{C}$. getaucht, darauf beide herausgenommen, rasch mit Fliesspapier sorgfältig getrocknet und nun mit gleichen, mittelstarken, tetanisirenden Strömen ${ }^{1}$ ) gereizt, so sieht man schon sehr bald reichliche Schweisströpfchen auf der gewärmten Seite auftreten, während die abgekühlte vollständig trocken bleibt, oder doch höchstens nur geringe Spuren von Secret liefert. Erst bei stärksten Strömen wird auch auf der abgekühlten Seite die Secretion etwas stärker.

Wird eine Katzenpfote, deren Secretionsnerv durchschnitten, durch einige Zeit (ca. $10 \mathrm{Min}$.) in Eiswasser gehalten, darauf sorgfältig getrócknet und nun local durch Einstich mit ca. $1 / \mathrm{g}$ ccm einer Pilocarpinlösung von $2 \%$ vergiftet, so tritt zwar bisweilen allerdings noch Secretion auf, aber doch stets in beträchtlich geringerem Grade wie auf einer zu $30^{\circ}$ erwärmten, mit gleicher Dosis beschickten Controlpfote.

In den mit Hrn. Trümpy zusammen angestellten Versuchen sahen wir fast momentan, nachdem wenige Tropfen einer concentrirten Pilocarpinlösung in die Ulnarseite des Handballens injicirt waren, Schweiss im Bereich dieser Einstichstelle auftreten und in reichlicher Menge sich steigern; hält nun aber die Versuchsperson den Ballen der Hand vorher für ca. 5-7 Min. in Eiswasser und macht man dann einen Einstich mit gleicher Menge gleich concentrirter Pilocarpinlösung, so trat oft erst nach mehr wie 6-8 Min. eine geringe Secretion ein.

b. Versuche über den Einfluss der Ueberhitzung auf die Erregbarkeit der Schweissdrüsen. Hält man an heissem Sommertage die eine Hand für ca. $10 \mathrm{Min}$. in Brunnenwasser von ca. 45-50 C., die andere zur Controle in. Wasser von $15-30^{\circ} \mathrm{C}$, trocknet dann beide Hände gut ab und macht rasch in der Hitze einen kleinen Spaziergang, so sieht man schon nach kïrzester Zeit an den Fingerspitzen, sowie an den Ballen der Vola auf der Controlseite reichliche Schweisströpfchen erscheinen, während 5, ja oft volle 10 Minuten verstreichen, bis auch die überhitzte, stark hyperämische Hand mit einer, noch einige Zeit nur spärlich erfolgenden Secretion anhebt. Gleichwohl sind aber die Schweissdrüsen durch unsern. Eingriff keineswegs etwa zu grösstem Theil in völlige Wärmestarre gebracht, denn schon ca. 30 -40 Minuten nach dem heissen Bade ist bei einem nochmaligen Schwitzversuche die Secretion auf beiden Seiten wieder in gleicher Stärke vorhanden.

Für das Ergebniss ist es gleichgültig, ob das Brunnenwasser durch verdünnte oder gesättigte Kochsalzlösung oder durch Schwefelsäure von $5 \%$ ersetzt war.

Mit stets gleichem Frfolg ist der. Versuch zu oft wiederholten Malen mir selbst sowie mehreren meiner Freunde geglückt.

1) Schlittenapparat von du Bois-Reymond, ein Daniell im primären Kreis, die Rollen berühren sich. 
Die Erregbarkeit der Schweissdrüsen als Function ihrer Temperatur. 481

Die angefiuhten Beobachtungen und Versuche liefern eine volle Bestätigung für die Eingangs angestellte Betrachtung. Zu hohe wie zu tiefe Temperaturen hatten die Schweissdrüsen bei Mensch und Katze in einen vorübergehenden Zustand der Lähmung versetzt. Die am Menschen gewonnenen Resultate waren durchaus schlagend, die Lähmung war nach beiden Eingriffen vollständig. Die Versuche an den Katzenpfoten zeigten ebenfalls recht deutlich den erwarteten Sinn der Erregbarkeitsänderung; wenn sie auch in Bündigkeit des Resultates ${ }^{1}$ ) um etwas hinter den vom Menschen gelieferten Ergebnissen zurïckblieben, so mag der Grund dieses Verhaltens ohne Fehl in der tiefern und desshalb vor Temperaturschwankungen geschitzteren Lage jener Schweissdrüsen zu suchen sein. -

Nunmehr scheinen wir endlich dem Verständniss einer Reihe älterer, noch unerklärter Beobachtungen näher getreten zu sein. In seinen epochemachenden Untersuchungen zur thierisehen Electricität Band II, 2, pag. 206-212 handelt du Boi s-Reymond von dem electromotorischen Verhalten ungleich erwärmter, sonst gleichartiger Hautstellen gegen einander.

„Folgendes war das Ergebniss meiner Versuche. Ein Finger bei $0^{\circ}$, worunter ich einen solchen verstehe, der einige Zeit in Lösung von $0^{0}$ verweilt hat, verbält sich so stark positiv gegen einen Finger bei $15^{\circ}, 30^{\circ}, 45^{\circ}$, dass die Nadel an die Hemmung gefuhrt wird. Die Wirkung ist aber bei $15^{\circ}$ oder $30^{\circ}$ änsserer Temperatur des zweiten Fingers weit heftiger als bei $45^{\circ}$. Ein Finger bei $15^{\circ}$ verhält sich gegen einen Finger bei $30^{\circ}$ schwach positiv. Gegen einen Finger bei $45^{\circ}$ dagegen verhält sich ein Finger bei $15^{\circ}$ oder bei $30^{\circ}$ sehr stark negativ. Am negativsten ist also der Finger bei etwa $30^{\circ}$. Bei jeder höheren sowohl als jeder tieferen Temperatur ist er positiver. Seine Positivität wächst nach beiden Riehtungen hin anfangs langsam, in der Nähe des Nullpunets und zwischen $40^{\circ}$ und $50^{\circ}$ aber ausserordentlich schnell."

Schon $d \mathfrak{u}$ Boi s selbst erwies die Vitalität der Erscheinungen; denn Leichenhände zeigten in entsprechenden Versuchen, dass ste ts der kältere Finger positiv gegen den wärmeren seí; damit konnte

1) Vergl. die entsprechenden Pilocarpinversuche. 
die Ursache des fraglichen Phänomens also namentlich nicht etwa auf blossen Thermoströmen beruhen.

Die nahen Beziehungen in welche unsere Beobachtungen zu dem eben ausgesprochenen Gesetze treten, sind sofort einlenchtend. Beides - die electromotorischen Kräfte, sowie das Schweissvermögen der Haut - sind gleichlaufende Functionen der Temperatur. Es liegt nahe, an eine causale Verknüpfung der beiden Abhängigveränderlichen za denken.

Erst nenlich noch hat eine von Hermann und mir ${ }^{1}$ ) gemeinschaftlich ausgeführte Untersuchung die secernirenden Schweissdrüsen der Katze als Sitz electromotorischer Kräfte dargethan. Schon geringe, kaum sichtbare Secretion auslösende Reize waren mächtig genug, recht erhebliche Ausschläge des stromprüfenden Magneten zu bewirken. - Wíe nan, wenn in den Versuchen von du Bo is eine, wenn auch nur geringe Schweisssecretion ebenfalls mitgespielt hätte? Auch ganz abgesehen von jeder Berücksichtigung der eben herrschenden Witterung, event. Jahreszeit (bei $d u$ Bois finden sich keine darauf bezügliche Angaben) dürfte allein schon die blosse, einer erwarteten Erseheinung zugewandte Aufmerksamkeit des Experimentators genügen, die Schweisscentren in gewisse Erregung zu bringen; dieselbe noch erheblich zu steigern, wird die starke sensible Reizung der Haut durch fast unerträgliche Kälte oder durch kaum ausstehliche Hitze wohl nicht verfehlen. Eine beiderseitig allerdings gleich starke centrale Erregung wird aber auf die Drüsen beider Seiten gemäss ihrer durch die verschiedene Temperatur bedingten verschiedenen Erregbarkeit in ganz verschiedenem Grade einwirken, eine verschieden starke Action der symmetrischen Drüsen wird demnach auch verschieden starke, in dem Leitersysteme einander entgegengesetzt gerichtete, electrische Ströme hervorrufen, die Richtung des stärkeren musste sich in den Versuchen von $\mathrm{d} \mathbf{u} \mathrm{B}$ o is zeigen. In der That lässt sich aus unsern Daten und Voraussetzungen eine Stromrichtung ableiten, wie sie in den Versuchen jenes Forschers stets zutraf. -

Genau gleich wie die Schweissdrüsen scheinen sich auch die Gefässe der Haut extremen Temperaturen gegeniiber zu verhalten. Die starke Hyperämie der erhitzten Hand leitet sich wenigstens aufs ungezwungenste aus einer Wärmelähmung der Hautarterien,

1) Diess Archiv Bd. XVII. p. 310-319. 
ab, die starke Röthung der Haut, wie solche in kalter Jahreszeit nach kurz vorübergehendem Blauwerden aufzutreten pflegt, zeigt die entsprechende Wirkung der Kältelähmung. Für die Beurtheilung gewisser neuerer Versuche über die Innervation der Gefässe ergibt sich so allerdings ein anderer Gesichtspunct. In solchem Sinne unternommene, neue Versuche haben bisher aber ihren $\mathrm{Ab}$ schluss noch nicht gefunden, wesshalb ich auf eine spätere Mittheilung verweisen muss.

(Aus dem physiologischen Laboratorium in Zürich.)

\section{Zum Verlauf der Schweissnerven der Katze.}

$$
\text { Von }
$$

\section{B. Luchsinger.}

Im Band XIV dieses Archivs machte ich die Angabe, dass sämmtliche Schweissfasern für die Hinterpfote der Katze am Oberschenkel in dem Strang des Hüftnerven verlaufen und fügte noch hinzu, dass diese jedoch gleichwohl dem Ischiadicas nicht von Ursprung an zugehörten, vielmehr insgesammt demselben erst aus dem Bauchstrang des Sympathicus zugeführt worden seien.

Gleichzeitig und unabhängig davon kam Ostroumoff ${ }^{1}$ ) zu vollständig gleichem Ergebniss.

Kurze Zeit hernach berichtete Nawrocki ${ }^{2}$ ) ebenfalls von völlig übereinstimmenden Versuchen.

An den Vorderpfoten waren die Schweissfasern schon früher in den Bahnen des Ulnaris und Medianus gefunden. Beinahe gleichzeitig und jedenfalls unabhängig von einander bezeichneten $\mathrm{Naw}$ rocki ${ }^{3}$ ) und $i c h^{4}$ ) ibereinstimmend den Bruststrang, spez. den Sternknoten des Sympathicus als alleinigen Durchgang. -

1) Moskaner ärztlicher Anzeiger 1876. (Russisch.) Vgl. Jahresbericht von Hofmann und Schwalbe. 1876.

2) Centralblatt für die med. Wissenschaften. 1878. Nr. 1.

3) Centralblatt für die med. Wissenschaften. 1878. Nr. 2.

4) Centralblatt für die med. Wissenschaften. 1878. Nr. 3. 